\title{
DOUBLE SCATTERING OF THE PLASMA STREAMS IN A BI-THERMAL IONOSPHERE
}

\author{
V. C. LIU and R. J. HUNG \\ Department of Aerospace Engineering, The University of Michigan, Ann Arbor, Michigan
}

(Received in final form 5 January, 1968)

\begin{abstract}
The plasma streams, emerged from their interactions with large bodies, are studied using the collisionless Boltzmann equation and the Poisson equation. The ambient ionosphere is assumed bithermal namely $T_{\mathrm{c}} / T_{1}=\alpha$, a constant. Numerical results are obtained with the ionospheres in thermal equilibrium $(\alpha=1)$ and quasi-equilibrium $(\alpha=10)$ for comparison. The velocity of the moving bodies is much larger than the thermal speed of the ions and much smaller than that of the electrons. Both cases, with and without the presence of a permanent magnetic field, are considered. The magnetic field is aligned with the motion of the bodies.
\end{abstract}

\section{INTRODUCTION}

The electro-gasdynamic interaction between a charged body and the plasma tail of another moving body in a tenuous plasma presents itself in many occasions. For illustrations, one can cite the case of a plasma probe which is placed in the wake of an orbiting satellite, the motion of a meteorite in a meteoric shower and the interaction of the solar wind with a comet tail (Biermann and Lüst, 1964). Among these cited examples, a common feature exists, namely, a plasma stream which has been slightly disturbed is interacting with the object of interest. In fact, the disturbed, rather than the quiescent, plasma stream is of more common occurrence in a physical environment, e.g., the ionosphere. To make our problem definite we postulate that the disturbed stream has been scattered from a neighboring object which is located upstream from the body of interest. This specific mode of interaction is referred here as the double scattering.

To further specify the problem, we assume that the ion mean free path $(\lambda)$ of the ambient plasma is many orders larger than the sizes $(R)$ of the bodies which, in turn, are large compared with the Debye length $(d)$ of the plasma, a characteristic length of the allowable polarization of the medium. It is also assumed that the velocity of the bodies $(V)$ is mesothermal (Liu, 1967), i.e., it is much larger than the thermal speed of the ions; smaller, than that of the electrons. These assumptions comply with most of the physical conditions imposed on the current problems of ionospheric gasdynamics e.g., the motion of a satellite in the ionosphere. The study of the plasma disturbances in the close vicinity of a moving body has been discussed elsewhere (Liu, 1967; Al'pert, Gurevich, and Pitaevskii, 1965; Liu and Jew, 1966; Liu, 1965). The theory of plasma far-wake behind a single body is also available (Al'pert, Gurevich, and Pitaevskii, 1965) though not complete. Our first objective is to develop a theory of the double scattering. We start with the single scattering i.e., the study of the far-wake behind a single body. A second motivation of the present work is to investigate the effect of the bi-thermal characteristics* of the ionosphere upon the theories of single and double scatterings. This has been found surprisingly strong when

* It has been often suggested on the basis of experimental evidence that the upper ionosphere could be in a quasi-equilibrium state having its electron temperature $\left(T_{e}\right)$ much higher than its ion temperature $\left(T_{1}\right)$. 
compared with the corresponding results for an ionosphere in thermal equilibrium $\left(T_{\mathrm{e}}=T_{1}=T\right)$.

Generally speaking, the mathematical problem of the present plasma interaction would entail the simultaneous solutions of the collisionless Boltzmann equation and the Maxwell equations of electromagnetics because the kinetic and the electromagnetic aspects of the interaction are coupled. This, indeed, is a hopeless task. It is found, however, by using inferences drawn from the assumed conditions of the interaction, much simplifications can be obtained. It is of interest to view the interaction in question from the standpoint of wave excitations by the moving bodies. It is well known (Stix, 1962) that there are four principal types of plasma waves in an ionized gas: (1) electromagnetic waves, (2) magnetohydrodynamic waves, and (3) electrostatic waves of electrons and of ions. With the assumed velocity for the moving body which is many orders smaller than the propagation speed of the electromagnetic waves, it is expected that the generation of electromagnetic waves by the bodies is insignificant. Among the many modes of the magnetohydrodynamic waves, we may consider the simplest one, the Alfven wave, which has a speed of propagation given by $H_{0} / \sqrt{ } 4 \pi m n_{0}$, where $m n_{0}$ is the mass density of the plasma; $H_{0}$, the magnetic field. For the upper ionosphere at $F$-max, the Alfven speed is estimated to be about $300 \mathrm{~km} / \mathrm{sec}$ which again is an order higher than the characteristic body velocity, e.g., the satellite velocities. Thus the excitation of the Alfven waves by the moving body would be negligible (Drell, Foley and Ruderman, 1965); it follows when the other modes of the magnetohydrodynamic waves are considered. Among the remaining types, the clectrostatic electron waves have a much higher speed than the bodies, as assumed in the present study, hence is not expected to be significantly excited. The electrostatic ion waves, also called ion-acoustic waves, which propagate with a velocity near the mean thermal speed of the ions, if $T_{\mathrm{e}} \approx T_{1}$, may become strongly excited by the moving bodies of interest. Indeed it will be seen that the plasma far-wake behind a moving body is characterized by the ionacoustic wave parameter, in appearance, much like the Mach wave in the continuum gasdynamics of neutral particles by the neutral acoustic waves. In view of these considerations, the Maxwell equations of electromagnetics, when applied to the present plasma interaction problem can be reduced to a single equation, the Poisson equation.

\section{MATHEMATICAL FORMULATION OF THE INTERACTION PROBLEM}

Consider two axially symmetric bodies with characteristic dimensions $R_{1}$ and $\boldsymbol{R}_{2}$ $\left(R_{1} \geq R_{2}\right)$ respectively at a distance $D\left(D \gg R_{1}\right)$ apart. A constant uniform, fully ionized gas, with velocity $\mathbf{V}$, flows past the bodies such that the velocity vector $\mathbf{V}$ is aligned with the axis of symmetry of the tandum bodies. A constant and uniform external magnetic field $(\mathrm{H})$ also aligns with the vector $\mathrm{V}$ (see Fig. 1). It is assumed that the (ion) mean free

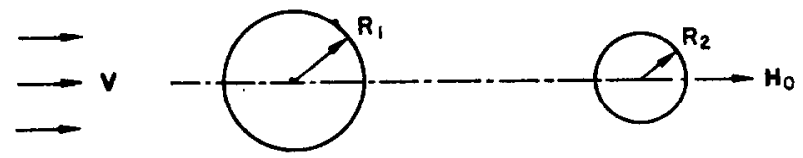

Fig. 1. TANDEM ARRANGEMENT OF THE TWO BODIES WITH ALIGNED MAGNETIC FIELD AND FREE STRFAM VEIOCTTY.

path of the ambient plasma is many orders larger than the ionic Larmor radius which, in turn, is much larger than the characteristic dimensions of the bodies while the Debye length is small compared to any of them. The free stream speed is assumed mesothermal which implies that it lies between the thermal speeds of ions and electrons and differs 
considerably from either of them. Under these conditions we would expect that the electron distribution of the disturbed flow will deviate negligibly from the MaxwellBoltzmann distribution, the ionic flow will exhibit the 'hypersonic' characteristics and yet the separation of the ion and electron species of the medium must not exceed a distance of the order of Debye length.

It should be further noted that in the present study of the plasma trail scattering, we are primarily concerned about the disturbances situated at large distances from the body where the field $(\phi)$ effect is small compared with the thermal effect. Under such an approximation, the terms representing the space charges in the Poisson equation may be written in terms of their ambient values plus a first order perturbation. The use of the small perturbation technique helps to linearize the system and thus the general principle of superposition becomes available. We shall regard the double-scattering effect as the superposition of quasi-single scattering effects.

In treating the far wake of the first obstacle $\left(R_{1}\right)$ to which the coordinate system is fixed we may determine the ion distribution $f$ by using the collisionless Boltzmann equation (also known as the Vlasov equation)

$$
\begin{aligned}
\mathbf{v} \cdot \frac{\partial f^{(1)}}{\partial \mathbf{r}}-\frac{\partial \phi}{\partial \mathbf{r}} \cdot \frac{\partial f_{0}}{\partial \mathbf{v}}+\frac{e}{m c}(\mathbf{v}+\mathbf{V}) \times \mathbf{H}_{0} \cdot & \frac{\partial f^{(1)}}{\partial \mathbf{v}} \\
& =\left\{\begin{array}{cc}
-\frac{\mathbf{r} \cdot \mathbf{V}}{|r|} f_{0} \delta\left(r-R_{1}\right) & \text { for } \mathbf{r} \cdot \mathbf{V}<0 \\
0 & \text { for } \mathbf{r} \cdot \mathbf{V}>0 .
\end{array}\right.
\end{aligned}
$$

Here $f^{(1)}=f-f_{0}$, the small disturbance of ion distribution deviated slightly from that of the Maxwellian $f_{0}$; where $v, c$ denote particle velocity and the speed of light respectively; $m$, the mass of an ion which is assumed singly charged with an amount $\mathrm{e}$. It is assumed that the effect of the induced magnetic field is negligible compared with either that of the electrostatic field or external magnetic field $\mathbf{H}_{0}$. The term on the right hand side of Equation (1) acts as a sink of the free stream ions $\left(f_{0}\right)$ neutralized by the surface $\left(R_{1}\right)$.

The electrostatic field in far wake stream is governed by the Poisson equation

$$
\nabla^{2} \phi=-4 \pi \mathrm{e}\left(n^{(1)}-n_{0} \frac{\mathrm{e} \phi}{K T \mathrm{e}}\right) \text {, }
$$

where $n^{(1)}=n-n_{0}$ denotes the small disturbance of ion number density deviated from that of the ambient ion density $n_{0}$.

We obtain the following equations as the Fourier components of Equations (1) and (2) for a spherical obstacle of radius $R_{1}$ with the coordinate system shown in Fig. 2.

$$
\begin{gathered}
\frac{\mathrm{e}}{m c}\left(\mathbf{u} \times \mathbf{H}_{0}\right) \cdot \frac{\partial f^{(\mathbf{1})}}{\partial \mathbf{u}}+\mathbf{i k} \cdot(\mathbf{u}-\mathbf{v}) f_{k}^{(1)}+\mathbf{i k} \cdot \mathbf{u} \frac{\mathrm{e} \phi_{k}}{K T_{i}} f_{0}=-\pi R_{1}{ }^{2} V f_{0} G \\
k^{2} \phi_{k}=-4 \pi \mathrm{e}\left(n_{k}^{(1)}-n_{0} \frac{\mathrm{e} \phi_{k}}{K T_{\mathrm{e}}}\right)
\end{gathered}
$$

Here

$$
\begin{gathered}
f_{k}^{(1)}=\int f^{(1)} \exp (-i \mathbf{r} \cdot \mathbf{k}) \mathrm{d} \mathbf{r}, \quad \phi_{k}^{(1)}=\int \phi \exp (-i \mathbf{r} \cdot \mathbf{k}) \mathrm{d} \mathbf{r}, \\
n_{k}^{(1)}=\int n^{(1)} \exp (-i \mathbf{r} \cdot \mathbf{k}) \mathrm{d} \mathbf{r} \mathbf{u}=\mathbf{v}+\mathbf{V}
\end{gathered}
$$


and

$$
G\left(k R_{1}, x\right)=\int_{0}^{\pi} \sin \theta \exp \left(-i k R_{1} \cos \theta \cos x\right) J_{0}\left(k R_{1} \sin \theta \sin x\right) \mathrm{d} \theta
$$

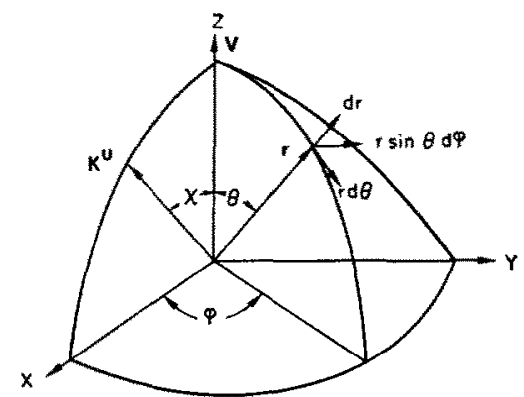

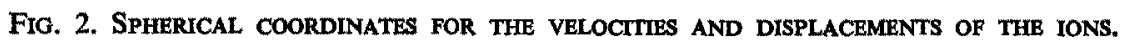

Note that the function $G\left(k R_{1}, x\right)$ approaches unity as $k R_{1}$ approaches zero; the latter condition represent the state of the far wake. In the present study the $k^{2}$ and higher order terms are neglected, thus Equations (3) and (4) can be readily reduced to

$$
\begin{gathered}
\frac{\mathrm{e}}{m c}\left(\mathbf{u} \times \mathbf{H}_{0}\right) \cdot \frac{\partial f_{k}^{(1)}}{\partial \mathbf{u}}+\mathbf{i k} \cdot(\mathbf{u}-\mathbf{V}) f_{k}^{(1)}+\mathbf{i k} \cdot \mathbf{u} \frac{\mathrm{e} \phi_{k}}{K T_{i}} f_{0}=-\pi R_{1}^{2} V f_{0}, \\
n_{k}^{(1)}=n_{0} \frac{\mathrm{e} \phi_{k}}{K T_{\mathrm{e}}} .
\end{gathered}
$$

Substituting Equation (6) into Equation (5), we have finally

$$
\frac{\mathrm{e}}{m c}\left(\mathbf{u} \times \mathbf{H}_{0}\right) \cdot \frac{\partial f_{k}^{(1)}}{\partial \mathbf{u}}+\mathbf{i k} \cdot(\mathbf{u}-\mathbf{V}) f_{k}^{(1)}+\mathbf{i} \alpha \mathbf{k} \cdot \mathbf{u} \frac{f_{0}}{n_{0}} n_{k}^{(1)}=-\pi R_{1}^{2} V f_{0},
$$

where the temperature ratio $\alpha=T_{\mathrm{e}} / T_{1}$.

\section{PLASMA SCATTERINGS WITHOUT MAGNETIC FIELD}

In many planetary plasma studies, it is justifiable to ignore the influence of the geomagnetic field. The neglect of the magnetic field effect from Equation (1) not only simplifies the analysis but also helps to focus the attention on the electrostatic aspect of the interaction. In the following study of the plasma trails scattered from the obstacles, we shall start with the single scattering which constitutes a preliminary step to the double scattering according to the present approach.

\subsection{Single scattering-far-wake behind the first obstacle}

It is recognized that the ion density in the far-wake behind an obstacle has been determined in previous works (Al'pert, Gurevich, and Pitaevskii, 1965) for an isothermal plasma $(\alpha=1)$. We shall follow essentially the same procedure but study for a general bi-thermal plasma where $\alpha=$ constant. It is also worthy to note that an additional ion density (rarefaction) lobe is found which was not reported in the previous works. Inasmuch as the procedure is similar to that in Al'pert, Gurevich, and Pitaevskii (1965), we shall refer to the latter for some of the mathematical details leading to the pertinent results of interest. 
From the formal solution to the perturbation Equation (7) for $f_{k}^{(1)}$ in Fourier components, we can obtain the first order deviation of the ion density from its ambient value, after integrations with respect to the full-range velocity components,

$$
n_{k}^{(1)}=\frac{i \pi R_{1}^{2} V \frac{1}{k} \int \frac{f_{0}}{u-V \cos x} \mathrm{du}}{1+\alpha+\frac{\alpha V \cos x}{n_{0}} \int \frac{f_{0}}{u-V \cos x} \mathrm{du}}
$$

and in terms of the Hilbert transform of Gaussian (Faddeyeva and Terentev, 1954), which is defined as

$$
Z(A)=\frac{1}{\pi \mathrm{i}} \int_{-\infty}^{+\infty} \frac{\exp -u^{2}}{u-A} \mathrm{~d} u=\exp -A^{2}\left(1+\frac{2 \mathrm{i}}{\sqrt{ } \pi} \int_{0}^{A} \exp \left(t^{2}\right) \mathrm{d} t\right) .
$$

Equation (8) can be written

$$
n_{k}^{(1)}=\pi R_{1}^{2} A^{2} \frac{n_{0}}{k} \Phi^{(1)}(A \cos x),
$$

where

$$
\Phi^{(1)}(A \cos x)=\frac{Z(A \cos x)}{1+\alpha+\mathrm{i} \alpha A \cos x Z(A \cos x)}
$$

and $A$ denotes the ion speed ratio $V /\left(2 K T_{1} / m\right)^{1 / 2}$.

The ion density perturbation in the Fourier components given in Equation (9) which is equal to the electron density perturbation under the present approximation, has been useful in determining the radar cross section for the back scattering of the electromagnetic waves in the determination of the trajectory of a re-entry body.

The inverse Fourier transform of Equation (9) gives the desired ion density variation in the physical space. The integration has been performed using the method of analytical continuation and Landau's contour of integration (Landau, 1946)

where

$$
n^{(1)}(r, \theta, A, \alpha)=-\frac{\pi R_{1}^{2} A^{2} n_{0}}{(2 \pi)^{2} r^{2}} F^{(1)}(\theta, A, \alpha),
$$

$$
\begin{gathered}
F^{(1)}(\theta, A, \alpha)=\cos \theta\left[\operatorname{Re} \Phi^{(1)^{\prime}}(A \sin \theta) \ln \sin \theta+\int_{\sin \theta}^{1} \operatorname{Re} \Phi^{(1)^{\prime \prime}}(A t) \ln \left[t+\left(t^{2}-\sin ^{2} \theta\right)^{1 / 2}\right] \mathrm{d} t\right. \\
\left.+\left\{\frac{\pi}{2} \operatorname{Im} \Phi^{(1)^{\prime}}(A \sin \theta)-\int_{0}^{\sin \theta} \operatorname{Im} \Phi^{(1)^{\prime \prime}}(A T) \sin ^{-1}\left(\frac{t}{\sin \theta}\right) \mathrm{d} t\right\}\right] \\
\Phi^{(1)}(A t)=\frac{\exp \left(-A^{2} t^{2}\right)}{[1+\alpha-2 \beta(A t) \gamma(A t)]^{2}+[\sqrt{ } \pi \beta(A t)]^{2}}\{\sqrt{ } \pi(1+\alpha) \\
+\mathrm{i}[-\pi \beta(A t)+2 \gamma(A t)\{1+\alpha-2 \beta(A t) \gamma(A t)\}]\} \\
\quad \Phi^{(1)^{\prime}}=\frac{\mathrm{d} \Phi^{(1)}}{\mathrm{d} t}, \quad \Phi^{(1)^{\prime \prime}}=\frac{\mathrm{d}^{2} \Phi^{(1)}}{\mathrm{d} t^{2}}, \\
\beta(A t)=\alpha A t \exp \left(-A^{2} t^{2}\right), \quad \gamma(A t)=\int_{0}^{A t} \exp \left(x^{2}\right) \mathrm{d} x
\end{gathered}
$$


where, $\operatorname{Re}$ and $\operatorname{Im}$ denote real and imaginary parts of a function respectively (for details see the Appendix).

Equation (12) has been evaluated by numerical analysis with a digital computer for the cases: (i) speed ratios $A=4$, temperature ratios $\alpha=1$; (ii) $A=4, \alpha=10$; (iii) $A=8, \alpha=1$; and (iv) $A=8, \alpha=10$. The plottings of the functions $F^{(1)}(\theta, A, \alpha)$ in polar coordinates are shown in Figs. ${ }^{*} 3-6$. Except for the factor $1 / r^{2}$, the function $F^{(1)}(\theta, A, \alpha)$ gives the relative magnitude of the deviation of ion density, also of electron density, from

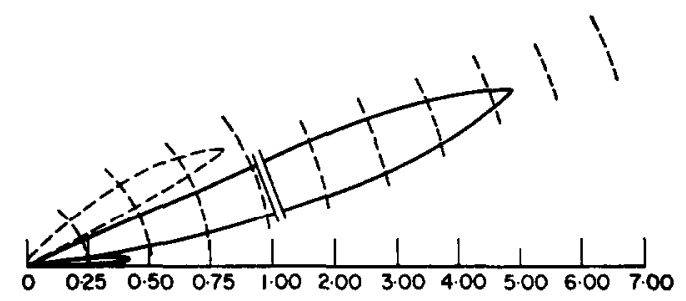

Fig. 3. Polar distribution of the ION-Density perturbation behind the first body. $n^{(1)}=n-n_{0}$ (speed ratio, $A=4$, temperature ratio $\alpha=1$ ).

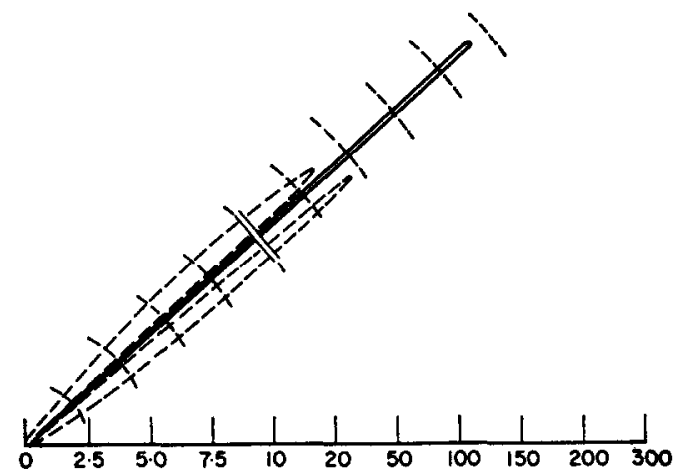

Fig. 4. POlar DISTRIBUtion OF THE ION-DENSTTY PERTURBation BehtND THE FIRST BOdY. $n^{(1)}=n-n_{0}(A=4, \alpha=10)$.

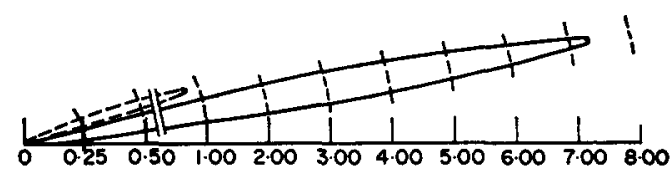

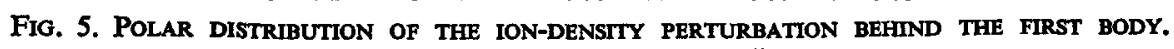
$n^{(1)}=n-n_{0}(A=8, \alpha=1)$.

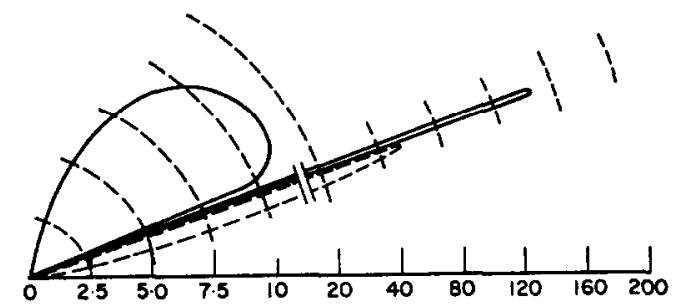

Fig. 6. Polar distribution of THE ION-DENSTTY PERTURbation beHIND THE FIRST BODY. $n^{(1)}=n-n_{0}(A=8, \alpha=10)$.

* Note the broken and segmented scales for the radial coordinates of the figures. 
the ambient density $n_{0}$ in the far wake as a function of angle $\theta$. The solid-curve denotes rarefraction $\left(n^{(1)}<0\right)$; dashed-curve, compression $\left(n^{(1)}>0\right)$. Notice that the maximum disturbance is reached on the surface of a cone with an included angle $2 \theta_{\max }$ where $\theta_{\max } \approx \sin ^{-1}\left(v_{1} / V\right)$ in the case where $\alpha=1$. This appears similar to the Mach cone in the continuum gasdynamics of neutral particles. The angle of the cone is determined by the ratio of the thermal velocity to the free stream velocity of the particles.

\subsection{Double scattering-far-wake behind the second obstacle}

Consider the plasma disturbance in the far wake of the second sphere $(D / r \ll 1)$ to which we now fix the coordinate system of Equations (1) and (2). Note that the incident stream to the second body $f_{01}=f_{0}+f^{(1)}$ which is proportional to $\exp \left\{-\left(m_{1} \mathbf{u}^{2}+2 \mathrm{e} \phi^{(1)}\right) /\right.$ $\left.2 K T_{i}\right\}$, and $\phi^{(1)}(r)$ is the field potential disturbance due to scattering of the first body. Replace, however, $f^{(1)}$ by $f^{(1)}+f^{(2)}$ and $f_{0}$ by $f_{01}$ in Equation (1). Again neglecting the magnetic field effect and the second order perturbation, as in (3.2), we obtain the increment in the ion density perturbation in the Fourier component, namely,

$$
n_{k}^{(2)}=\frac{\pi R_{1}^{2} A^{2} n_{0}}{k}\left[\sigma^{2} \Phi^{(1)}(A \cos x)-\Phi_{1}^{(2)}(A \cos x)\right]
$$

where

$$
\begin{gathered}
n_{k}^{(2)}=\int f_{k}^{(2)} \mathrm{du}, \quad \sigma=\frac{R_{2}}{R_{1}} \exp \left(-\mathrm{e} \phi_{R_{2}} / K T_{1}\right) . \\
\phi_{R_{\mathrm{z}}}=\text { electrostatic potential at } r=R_{2} \\
\left.\Phi_{1}^{(2)}=\frac{Z(A \cos x)}{[1+\alpha+\mathrm{i} \alpha A \cos x Z(A \cos x)]^{2}}\right] \text { with coordinate fix at the first body. }
\end{gathered}
$$

Following the same procedure as that in Section 3.1, we obtain the inverse Fourier transform as follows;

$$
\begin{gathered}
n^{(2)}(r, \theta, A, \alpha)=\frac{\pi R_{1}^{2} A^{2} n_{0}}{(2 \pi)^{3}}\left[\sigma^{2} \int_{0}^{\pi} \Phi^{(1)}(A \cos x) \sin x \mathrm{~d} x \int_{0}^{\infty} k \mathrm{~d} k \int_{0}^{2 \pi} \exp \{\mathrm{i} k r(\cos \theta \cos x\right. \\
+\sin \theta \sin x \cos \varphi)\} \mathrm{d} \varphi \\
-\int_{0}^{\pi} \Phi_{1}^{(2)}(A \cos x) \sin x \mathrm{~d} x \int_{0}^{\infty} k \mathrm{~d} k \int_{0}^{2 \pi} \exp \left\{\mathrm{i} k r\left[\left(\cos \theta+\frac{D}{r}\right) \cos x+\sin \theta \sin x \cos \varphi\right]\right\} \mathrm{d} \varphi \\
=-\frac{\pi R_{1}^{2} A^{2} n_{0}}{(2 \pi)^{2} r^{2}}\left[\sigma^{2} \cos \theta \int_{-1}^{1} \frac{\Phi^{(1)}(A t) t \mathrm{~d} t}{\left[t^{2}-\sin ^{2} \theta+2 \mathrm{i} \delta \cos x \cos \theta\right]^{3 / 2}}\right. \\
-\frac{\left(\cos \theta+\frac{D}{r}\right)}{\left(1+2 \frac{D}{r} \cos \theta+\frac{D^{2}}{r^{2}}\right)^{3 / 2}} \\
\left.\times \int_{-1}^{1} \frac{\Phi_{1}^{(2)}(A t) t \mathrm{~d} t}{\left[\left(1+2 \frac{D}{r} \cos \theta+\frac{D^{2}}{r^{2}}\right) t^{2}-\sin ^{2} \theta+2 \mathrm{i} \delta\left(\cos \theta+\frac{D}{r}\right) \cos x\right]^{3 / 2}}\right]
\end{gathered}
$$


where $t=\cos x$.

Notice that $\operatorname{Re} \Phi^{(1)}(A t)$ and $\operatorname{Re} \Phi_{1}^{(2)}(A t)$ are even functions; $\operatorname{Im} \Phi^{(1)}(A t)$ and $\operatorname{Im} \Phi_{1}^{(2)}(A t)$, odd functions. Letting $\delta \rightarrow 0^{+}$, we obtain (for details see the Appendix)

where

$$
n^{(2)}(r, \theta, A, \alpha)=-\frac{\pi R_{1}^{2} A^{2} n_{0}}{(2 \pi)^{2} r^{2}}\left[\sigma^{2} F^{(1)}(\theta, A, \alpha)-F_{1}^{(2)}(\theta, A, \alpha)\right]
$$

$$
\begin{aligned}
& F_{1}^{(2)}(\theta, A, \alpha)=\frac{\left(\cos \theta+\frac{D}{r}\right)}{\left(1+2 \frac{D}{r} \cos \theta+\frac{D^{2}}{r^{2}}\right)^{3 / 2}} \\
& \times\left[\operatorname{Re} \Phi_{1}^{(2)^{\prime}}\left(\frac{\sin \theta}{\sqrt{1+2 \frac{D}{r} \cos \theta+\frac{D^{2}}{r^{2}}}}\right) \ln \left\{\frac{\sin \theta}{\sqrt{1+2 \frac{D}{r} \cos \theta+\frac{D^{2}}{r^{2}}}}\right\}\right.
\end{aligned}
$$

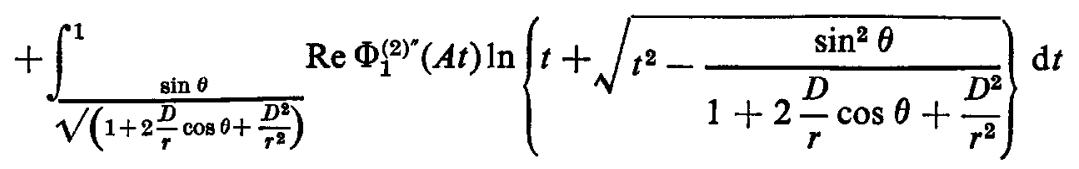

$$
\begin{aligned}
& +\int \frac{\pi}{2} \operatorname{Im} \Phi_{1}^{(2)^{\prime}}\left(\frac{\sin \theta}{\sqrt{1+2 \frac{D}{r} \cos \theta+\frac{D^{2}}{r^{2}}}}\right)
\end{aligned}
$$

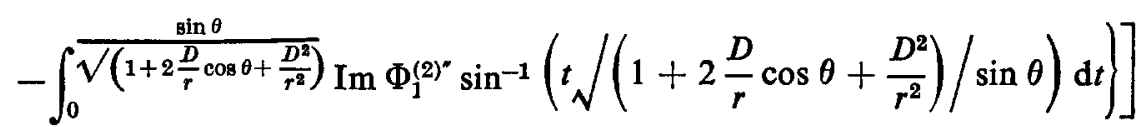

Under the assumption $D / r \ll 1$, Equation (17) simplifies to

where

$$
n^{(2)}(r, \theta, A, \alpha)=-\frac{\pi R_{2}{ }^{2} A^{2} n_{0}}{(2 \pi)^{2} r^{2}} F^{(2)}(\theta, A, \alpha),
$$

$$
\begin{aligned}
F^{(2)}(\theta, A, \alpha) & =\cos \theta\left[\operatorname{Re} \Phi^{(2) \prime}(A \sin \theta) \ln \sin \theta+\int_{\sin \theta}^{1} \operatorname{Re} \Phi^{(2) \prime \prime}(A t) \ln \left[t+\left(t^{2}\right.\right.\right. \\
& \left.+\left\{\frac{\pi}{2} \operatorname{Im} \Phi^{(2)^{\prime}}(A \sin \theta)-\int_{0}^{\sin \theta} \operatorname{Im} \Phi^{(2) \prime}(A t) \sin ^{-1}\left(\frac{t}{\sin \theta}\right) \mathrm{d} t\right\}\right] \\
& \Phi^{(2)}(A t)=\frac{\exp \left(-A^{2} t^{2}\right)}{\left[\xi^{2}(A t)-\pi \beta^{2}(A t)\right]^{2}+4 \pi[\xi(A t) \beta(A t)]^{2}}\left(\sqrt { } \pi \left[\left\{1+\alpha-\sigma^{-2}\right.\right.\right. \\
& -4 \beta(A t) \gamma(A t)\}\left\{\xi^{2}(A t)-\pi \beta^{2}(A t)\right\}+2 \alpha \exp \left(-A^{2} t^{2}\right) \xi(A t) \\
& \left.\times\left\{2 A t \gamma(A t)\left[\xi(A t)-\sigma^{-2}\right]+\pi A t \beta(A t)\right\}\right] \\
& +\mathrm{i}\left[\left\{\xi^{2}(A t)-\pi \beta^{2}(A t)\right\}\{\pi \beta(A t)+2 \gamma(A t)[\xi(A t)\right. \\
& \left.\left.-\sigma^{-2}\right]\right\}-2 \pi \beta(A t)\left\{1+\alpha-\sigma^{-2}-4 \beta(A t) \gamma(A t)\right\} \\
& \times \xi(A t)]\},
\end{aligned}
$$


where

$$
\Phi^{(2)^{\prime}}=\frac{\mathrm{d} \Phi^{(2)}}{\mathrm{d} t}, \quad \Phi^{(2)^{n}}=\frac{\mathrm{d}^{2} \Phi^{(2)}}{\mathrm{d} t^{2}}, \quad \xi(A t)=1+\alpha-2 \beta(A t) \gamma(A t) .
$$

The function $F^{(2)}(\theta, A, \alpha)$ has been evaluated numerically for the following cases: $A=4, \alpha=1 ; A=4, \alpha=10 ; A=8, \alpha=1 ; A=8, \alpha=10$. These are plotted in Figs. * 7-10. Except for the factor $1 / r^{2}, F^{(2)}(\theta, A, \alpha)$ gives the additional relative ion

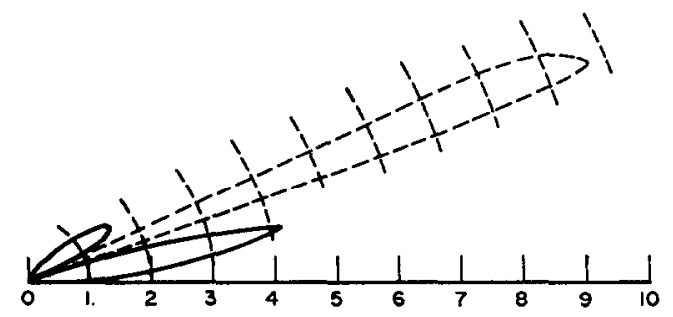

Fig. 7. Polar distribution of the PERTURbed iON-Density deViation Behind the SECOND BODY.

$$
\begin{gathered}
n^{(2)}=n-\left(n_{0}+n^{(1)}\right),(\text { speed ratio } A-4, \text { temperature ratio } \alpha=1, \\
\text { body size ratio } \left.\left(R_{1} / R_{2}\right)=10\right)
\end{gathered}
$$

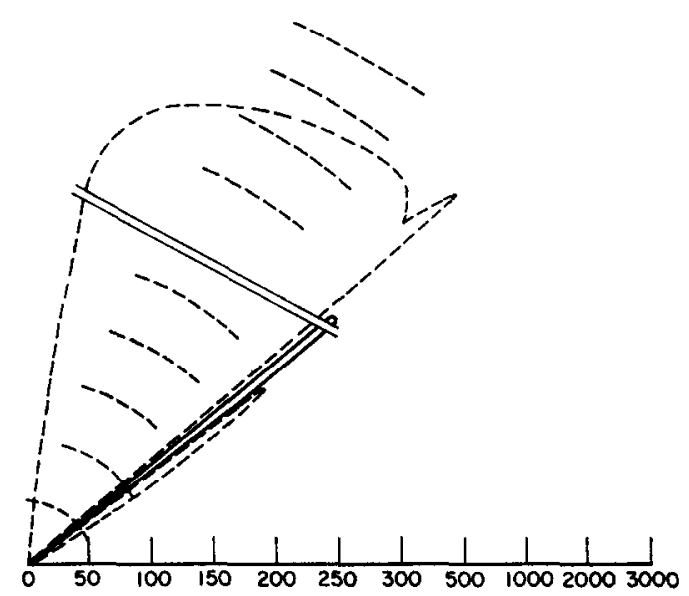

Fia. 8. Polar distribution Of THE PERTURBed ION-DENSITY DEVIATION BEHIND THE SECOND BODY.

$$
n^{(2)}=n-\left(n_{0}+n^{(1)}\right),\left(A=4, \alpha=10, R_{1} / R_{2}=10\right) .
$$

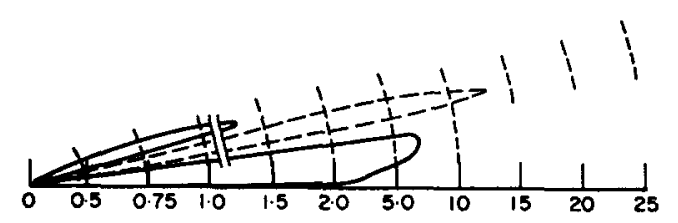

FiG. 9. POLAR distribution of THE PERTURBed ION-DENSTTY DEVIATION BEHIND THE SECOND

$$
n^{(2)}=\left(n-n_{0}+n^{(1)}\right), \stackrel{\text { BODY. }}{\left(A=8, \alpha=1, R_{1} / R_{\mathrm{a}}=10\right) .}
$$

* Note the broken and segmented scales for the radial coordinates of the figures. 


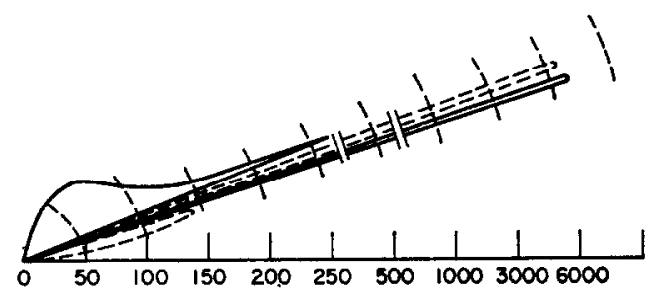

FIG. 10. POLAR DISTRIBUTION OF THE PERTURBED ION-DENSTTY DEVIATION BEHIND THE SECOND BODY.

$\left(A=8, \alpha=10, R_{1} / R_{2}=10\right)$.

disturbance as a function $\theta$, due to the presence of $f_{k}^{(1)}$ superposed to $f_{0}$ as the 'free stream' distribution for the second obstacle. These additional lobes in the ion density distribution are generated through the transformation of the Fourier components of $n_{k}^{(1)}$ by interaction with the second sphere. Hence the total disturbance of the ion density relative to its ambient value is given by $n^{(1)}(r, \theta, A, \alpha)+n^{(2)}(r, \theta, A, \alpha)$.

\section{PLASMA SCATTERINGS WITH A MAGNETIC FIELD}

When a permanent magnetic field $\left(H_{0}\right)$ is added, the motion of the charged particles will be under the dual influences of the electrostatic and the magnetic field as shown in Equation (1). It is the relative strength of the two that determines the motion of the particles. In the equilibrium state, electrons distribute according to the Maxwell-Boltzmann law. In the case where the magnetic field $\left(H_{0}\right)$ aligns with the free stream such as we have here, the disturbed electron velocity-distribution is not expected to maintain essentially isotropic with zero electrostatic field because the electrons will have more freedom to move in the direction of the magnetic field. This non-Maxwellian effect for the electrons will be ignored in the present study. It is assumed that the Larmor (ion) radius is large compared with the characteristic dimension of the bodies such that the sink term on the right hand side of Equation (1) will not be affected by the Larmor gyrations of the ions. Procedures similar to that of Section 3 will be followed to treat the plasma scatterings with a magnetic field.

\subsection{Single scattering-far-wake behind the first obstacle}

Starting with Equation (7) and letting the Larmor (ion) frequency $\Omega_{1}=\mathrm{e} H_{0} / m c$ and the coordinate system be fixed to the first sphere we have (see Fig. 11),

$$
\begin{aligned}
\frac{\partial f_{k}^{(1)}}{\partial \beta}-\frac{\mathrm{i}}{\Omega_{1}}\left(k_{\perp} u_{\perp} \cos \beta+k_{\|} u_{\|}-\mathbf{k}\right. & . \mathrm{V}) f_{k}^{(1)} \\
& +\frac{\mathrm{i} \alpha}{\Omega_{1}}\left(u_{\perp} k_{\perp} \cos \beta+k_{\|} u_{\|}\right) \frac{f_{0}}{n_{0}} n_{k}^{(1)}=\frac{\pi R_{1}^{2} f_{0}}{\Omega_{1}} .
\end{aligned}
$$

From the solution for $f_{k}^{(1)}$ which is then integrated with respect to the velocity components, $n_{k}^{(1)}$ is obtained.

$$
n_{k}^{(1)}=\frac{n R_{1}{ }^{2} n_{0} V}{\Omega_{1}} \frac{\Psi\left(k_{\|}, k_{\perp}\right)}{1+\alpha+\frac{i \alpha \mathbf{k} . \mathbf{V}}{\Omega_{1}} \Psi\left(k_{\|}, k_{\perp}\right)},
$$




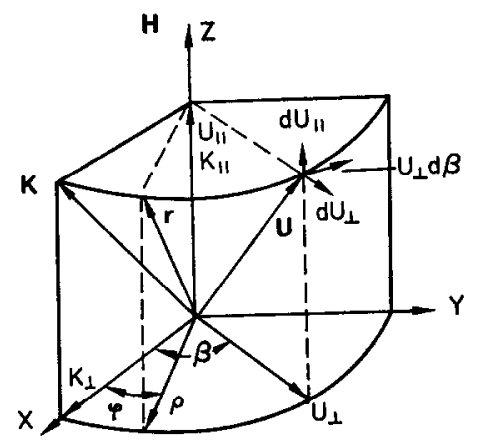

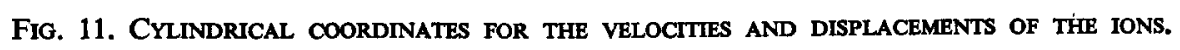
where

$$
\Psi\left(k_{\|}, k_{\perp}\right)=\int_{0}^{\infty} \exp \left[\frac{\mathrm{ik} \cdot \mathbf{V}}{\Omega_{1}} x-\frac{K T_{1}}{2 m \Omega_{i}^{2}}\left(k_{\|}^{2} x^{2}+4 k_{\perp}{ }^{2} \sin ^{2} \frac{x}{2}\right)\right] \mathrm{d} x .
$$

It is of interest to start with a trivial case by assuming a weak magnetic field such that $v_{1} k_{\|} / \Omega_{1} \gg 1$ where $v_{1}$ denotes the mean thermal speed of ions $\left(2 K T_{1} / m\right)^{1 / 2} ; k_{\|}$, the wave component parallel to the magnetic field $\left(H_{0}\right)$. Under this condition, it is noted that the integrand of the integral (24) becomes extremely simplified. Following the approximation introduced in Al'pert, Gurevich, and Pitaevskii (1965), we ignore the small contribution to the integral for $|x| \geq 1$ and approximate $\sin ^{2}(x / 2)$ by $x^{2} / 4$ for $|x|<1$;

$$
\Psi\left(k_{\|}, k_{\perp}\right)=\int_{0}^{\infty} \exp \left[\frac{\mathrm{i} \mathbf{k} \cdot \mathbf{V}}{\Omega_{\mathrm{I}}} x-\frac{K T_{\mathrm{i}}}{2 m \Omega_{\mathrm{i}}^{2}} k^{2} x^{2}\right] \mathrm{d} x,
$$

where $k^{2}=k_{\perp}^{2}+k_{\|}^{2}$. The integral (25) can be further simplified by the use of the identity:

$$
\int_{0}^{\infty} \exp \left(\mathrm{i} t b-\frac{t^{2}}{4}\right) \mathrm{d} t=\left(\sqrt{ } \pi+2 \mathrm{i} \int_{0}^{b} \exp \left(t^{2}\right) \mathrm{d} t\right) \exp \left(-b^{2}\right)=Z(b) \text {. }
$$

Hence

$$
\Psi\left(k_{\|}, k_{\perp}\right)=\frac{\Omega_{1}}{v_{1} k} Z(A)
$$

and

$$
n_{k}^{(1)}=\frac{\pi R_{1}^{2} A n_{0}}{k} \frac{Z(A)}{1+\alpha+\mathrm{i} \alpha A Z(A)} .
$$

Notice that this is identical to the result of Equation (9). Thus we have substantiated the validity of the non-magnetic approximation to the study of a planetary plasma provided the local geomagnetic field is sufficiently weak, i.e., $v_{1} k_{\|} / \Omega_{1} \gg 1$.

We shall proceed with the case of the opposite extreme where the magnetic field effect becomes a dominant influence by postulating

$$
\frac{v_{1} k_{\|}}{\Omega_{1}} \ll 1 .
$$

It is clear that under a dominant influence of magnetic field, the ions must behave such that the Fourier component of the ion density $n_{k}^{(1)}$ will have sharp maxima when values of 
$k_{\| 1} V / \Omega_{1}$ are close to integers. In other words, $n_{k}^{(1)}$ will have a Maximum value whenever $k_{\mathbb{l}} V$ equals an integral multiple of the ion cyclotron frequency $\Omega_{\mathbb{1}}$. Now let

$$
\frac{k_{\|} V}{\Omega_{1}}=N+\varepsilon,
$$

where $N=0, \pm 1, \pm 2 \ldots$ and $|\varepsilon|<1$, be introduced to Equation (24) which becomes

$$
\Psi\left(k_{\|}, k_{\perp}\right)=\int_{0}^{\infty} \exp \left(\mathrm{i} \varepsilon x-\frac{v_{1}^{2}}{4 \Omega_{1}^{2}} k_{\|}^{2} x^{2}\right) \exp \left(\mathrm{i} N x-\frac{v_{1}^{2}}{\Omega_{1}^{2}} k_{\perp}{ }^{2} \sin ^{2} \frac{x}{2}\right) \mathrm{d} x .
$$

An approximation to the above integral can be made by noting (Al'pert, Gurevich, and Pitaevskii, 1965) that the second exponential factor varies much more rapidly than the first under the strong field condition (29), hence the integral (31) can be approximated by replacing the rapidly varying factor with its averaged value over a cycle:

$$
\frac{1}{2 \pi} \int_{0}^{2 \pi} \exp \left(\mathrm{i} N x-\frac{v_{1}^{2}}{\Omega_{\mathrm{I}}{ }^{2}} k_{\perp}{ }^{2} \sin ^{2} \frac{x}{2}\right) \mathrm{d} x \approx \exp \left(-\frac{k_{\perp}{ }^{2} v_{\mathrm{I}}{ }^{2}}{2 \Omega_{\mathrm{I}}{ }^{2}}\right) \mathrm{I}_{n}\left(\frac{k_{\perp}{ }^{2} v_{\mathrm{i}}{ }^{2}}{2 \Omega_{\mathrm{I}}{ }^{2}}\right),
$$

where $\mathrm{I}_{n}$ denotes the Bessel function of an imaginary argument. Accordingly,

$$
\Psi\left(k_{\|}, k_{\perp}\right)=\frac{\Omega_{1}}{v_{1} k_{\|}} Z\left(\frac{\| V-N \Omega_{1}}{k_{\|} v_{1}}\right) \exp \left(-\frac{k_{\perp}^{2} v_{1}^{2}}{2 \Omega_{\mathrm{i}}^{2}}\right) I_{n}\left(\frac{k_{\perp}^{2} v_{i}^{2}}{2 \Omega_{\mathrm{I}}^{2}}\right)
$$

and

$$
n_{k}^{(1)}=\frac{\pi R_{1}^{2} n_{0} A}{k_{\|}} \frac{W\left(k_{\|}, k_{\perp}\right)}{1+\alpha+i \alpha A W\left(k_{\|}, k_{\perp}\right)},
$$

where

$$
W\left(k_{\|}, k_{\perp}\right)=Z\left(\frac{k_{\|} V-N \Omega_{1}}{k_{\|} v_{\mathrm{I}}}\right) \exp \left(-\frac{k_{\perp}^{2} v_{\mathrm{i}}^{2}}{2 \Omega_{\mathrm{I}}^{2}}\right) \mathrm{I}_{n}\left(\frac{k_{\perp}^{2} v_{\mathrm{I}}^{2}}{2 \Omega_{\mathrm{I}}^{2}}\right) .
$$

The inverse Fourier transform of $n_{k}^{(1)}$ given in (34) is performed by using a cylindrical wave space, $\mathrm{d} \mathbf{k}=k_{\perp} \mathrm{d} k_{\perp} \mathrm{d} k_{\|} \mathrm{d} \phi$ leading to

$$
n^{(1)}(\rho, z)=\frac{\pi R_{1}^{2} n_{0} A}{(2 \pi)^{3}} \int_{k_{\perp}=0}^{+\infty} \int_{k_{\|=-\infty}}^{+\infty} \frac{W\left(k_{\|}, k_{\perp}\right) J_{0}\left(\rho k_{\perp}\right)}{1+\alpha+i \alpha A W\left(k_{\|}, k_{\perp}\right)} \frac{k_{\perp} \mathrm{d} k_{\perp} \mathrm{d} k_{\|}}{k_{\|}},
$$

where $J_{0}$ is the Bessel function of the first kind.

Since the evaluation of the integrals in (36) depends on

$$
Z\left(\frac{k_{\|} V-N \Omega_{1}}{k_{\|} v_{\mathbf{1}}}\right)=Z\left(\frac{\varepsilon \Omega_{1}}{k_{\|} v_{1}}\right)
$$

which can be approximated for different frequencies, we shall discuss the two following cases:

(1) When the frequency of the perturbation wave, induced by the obstacle, is not close to the ion-cyclotron frequency $\Omega_{\mathrm{i}}$, such that,

$$
k_{\|} V-N \Omega_{1} \gg k_{\|} v_{1} \text { or } \varepsilon \Omega_{1} / k_{\|} v_{1} \gg 1
$$

expand the $Z$-function (37) (see Fried and Conte, 1961),

$$
z\left(\frac{k_{\|} V-N \Omega_{1}}{k_{\|} v_{\mathrm{i}}}\right) \approx \mathrm{i}\left(\frac{k_{\|} V-N \Omega_{\mathrm{i}}}{k_{\|} v_{\mathrm{i}}}\right)^{-1}
$$


the integrand of Equation (36) becomes

$$
\mathrm{i} \iint \frac{v_{\mathrm{i}} P\left(k_{\perp}^{2}\right) \exp \left(\mathrm{i} z k_{\|}\right) J_{0}\left(\rho k_{\perp}\right)}{(1+\alpha)\left(k_{\|} V-N \Omega_{\mathrm{j}}\right)-\alpha V k_{\|} P\left(k_{\perp}^{2}\right)} k_{\perp} \mathrm{d} k_{\perp} \mathrm{d} k_{\|},
$$

where

$$
P\left(k_{\perp}^{2}\right)=\exp \left(-\frac{k_{\perp}^{2} v_{1}^{2}}{2 \Omega_{1}^{2}}\right) I_{n}\left(\frac{k_{\perp}^{2} v_{1}^{2}}{2 \Omega_{1}^{2}}\right) .
$$

Note that the singularity of the integrand in (40) is located on the real axis, the contour of integration is chosen as shown in Fig. 12. After the integration of Equation (36) over

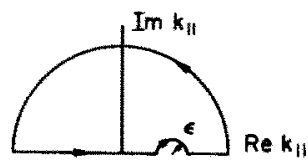

Fig. 12. Closed CONTOUR of nNTEGRATION.

$\mathrm{d} k_{\|}$is performed, we obtain finally

$$
n^{(1)}(\rho, z)=-\frac{\pi^{2} R_{1}^{2} n_{0}}{(2 \pi)^{3}} \mathrm{i} \int_{0}^{\infty} \frac{P\left(k_{\perp}^{2}\right) \exp \left\{\frac{i z(1+\alpha) N \Omega_{1}}{V\left[\left(1+\alpha-\alpha P\left(k_{\perp}^{2}\right)\right]\right.}\right\} J_{0}\left(\rho k_{\perp}\right) k_{\perp} \mathrm{d} k_{\perp}}{\left[1+\alpha-\alpha P\left(k_{\perp}^{2}\right)\right]} .
$$

(2) When the frequency of the perturbation wave, induced by the obstacle, is very close to the ion-cyclotron frequency $\Omega_{1}$, such that,

$$
k_{\|} V-N \Omega_{\mathrm{i}} \ll k_{\|} v_{\mathrm{i}} \text { or } \varepsilon \Omega_{\mathrm{i}} / k_{\|} v_{\mathrm{i}} \ll 1
$$

expand the $Z$-function (37) (see Fried and Conte, 1961),

$$
Z\left(\frac{k_{\|} V-N \Omega_{1}}{k_{\|} v_{1}}\right) \approx \mathrm{i} \pi^{1 / 2}\left\{1-\left(\frac{k_{\|} V-N \Omega_{1}}{k_{\|} v_{1}}\right)^{2}\right\} \approx \mathrm{i} \pi^{1 / 2}
$$

and Equation (36) is evaluated giving finally

$$
n^{(1)}(\rho, z)=-\frac{\pi^{5 / 2} R_{1}^{2} n_{0} A}{(2 \pi)^{3}} \times(\rho),
$$

where

$$
\mathrm{X}(\rho)=\int_{0}^{\infty} \frac{P\left(k_{\perp}^{2}\right) J_{0}\left(\rho k_{\perp}\right)}{1+\alpha-\pi^{1 / 2} \alpha A P\left(k_{\perp}^{2}\right)} k_{\perp} \mathrm{d} k_{\perp} .
$$

\subsection{Double scattering-far-wake behind the second obstacle}

In studying the disturbed field far down-stream from the second sphere, ${ }^{*}$ we follow the same rule of substitution as used in Section 3.1 with the coordinate system fixed to the second sphere. The relative order of the magnetic effect in the Equation (7) used under the condition $v_{1} k_{\|} / \Omega_{1} \ll 1$ for the first body will be maintained. We shall dispense with the study of the case with weak field because of its triviality.

- We use a similar assumption as in Section 3.2 , namely $D / z \ll 1$. 
Contrast to the non-magnetic case shown in Equation (15), we have

$$
n_{k}^{(2)}=n_{k}^{(1)}\left[\sigma^{2}-\frac{1}{1+\alpha+\frac{\mathrm{i} \alpha k_{\|} V}{\Omega_{i}} \Psi\left(k_{\|}, k_{\perp}\right)}\right]
$$

which, after inverse Fourier transform, becomes

$$
\begin{aligned}
& n^{(2)}(\rho, z)=\frac{\pi R_{2}{ }^{2} n_{0} A}{(2 \pi)^{3}} \int_{k_{\perp}=0}^{+\infty} \int_{k_{\|=-\infty}}^{+\infty} \frac{W\left(k_{\|}, k_{\perp}\right)\left[Q\left(k_{\|}, k_{\perp}\right)-\sigma^{-2}\right]}{Q^{2}\left(k_{\|}, k_{\perp}\right)} \exp \left(\mathrm{i} z k_{\|}\right) \\
& \times \frac{J_{0}\left(\rho_{\perp}\right)}{k_{\|}} k_{\perp} \mathrm{d} k_{\perp} \mathrm{d} k_{\|}
\end{aligned}
$$

where

$$
Q\left(k_{\|}, k_{\perp}\right)=1+\alpha+i \alpha A W\left(k_{\|}, k_{\perp}\right)
$$

The integral (48) is to be approximated under different frequencies of the perturbation waves in the same manner as was done in Section 4.1.

(1) $k_{\|} V-N \Omega_{\mathrm{i}} \gg k_{\|} v_{\mathrm{i}}$ or $\varepsilon \Omega_{\mathrm{i}} / k_{\|} v_{\mathrm{i}} \gg 1$.

Using the similar approximation for the $Z$-function as in (39) and the same contour (Fig. 12), the integration for integral (48) is performed.

$$
\begin{aligned}
n^{(2)}(\rho, z)=-\frac{\pi^{2} R_{2}{ }^{2} n_{0}}{(2 \pi)^{g} V} & \int_{0}^{\infty} \frac{P\left(k_{\perp}{ }^{2}\right) J_{0}\left(\rho k_{\perp}\right) \exp \left[\frac{\mathrm{i} n \Omega_{1}(1+\alpha) z}{V S\left(k_{\perp}{ }^{2}\right)}\right]}{S^{2}\left(k_{\perp}{ }^{2}\right)} \\
& \times\left[V\left\{S\left(k_{\perp}{ }^{2}\right)-\sigma^{-2}\right\}+\mathrm{i} n \Omega_{1} \sigma^{-2} z\left\{1-\frac{1+\alpha}{S\left(k_{\perp}{ }^{2}\right)}\right\}\right] k_{\perp} \mathrm{d} k_{\perp},
\end{aligned}
$$

where

$$
S\left(k_{\perp}{ }^{2}\right)=1+\alpha-\alpha P\left(k_{\perp}{ }^{2}\right)
$$

(2) $k_{\|} V-N \Omega_{1} \ll k_{\|} v_{1}$ or $\varepsilon \Omega_{1} / k_{\|} v_{1} \ll 1$.

Using the similar approximation for the $Z$-function as in (44), we obtain finally

where

$$
n^{(2)}(\rho, z)=-\frac{\pi^{5 / 2} R_{2}{ }^{2} n_{0} A}{(2 \pi)^{3}} Y(\rho)
$$

$$
\begin{aligned}
Y(\rho) & =\int_{0}^{\infty} \frac{P\left(k_{\perp}^{2}\right) J_{0}\left(\rho k_{\perp}\right)\left[T\left(k_{\perp}{ }^{2}\right)-\sigma^{-2}\right]}{T^{2}\left(k_{\perp}{ }^{2}\right)} k_{\perp} \mathrm{d} k_{\perp} \\
T\left(k_{\perp}{ }^{2}\right) & =1+\alpha-\pi^{1 / 2} \alpha A P\left(k_{\perp}{ }^{2}\right) .
\end{aligned}
$$

In the numerical computation of the integrals in (42) and (50), we choose the ionosphere parameters (Al'pert, Gurevich, and Pitaevskii, 1965) corresponding to an altitude of $1000 \mathrm{~km}$. It is estimated that for $N=1, k_{\perp}{ }^{2} v_{1}^{2} / 2 \Omega_{1}^{2} \sim 1.32 \times 10^{-2}$ and $\mathrm{I}_{n}\left(k_{\perp}^{2} v_{1}^{2} / 2 \Omega_{1}^{2}\right)$ $\sim 6.6 \times 10^{-8}$. Note that $1+\alpha \gg \alpha P\left(k_{1}{ }^{2}\right)$, Equations (42) and (50) reduce to the forms

$$
n^{(1)}(\rho, z)=-\frac{\pi R_{1}^{2} n_{0}}{(2 \pi)^{3}(1+\alpha)}\left(\mathrm{i} \cos \frac{\Omega_{1} z}{V}-\sin \frac{\Omega_{1} z}{V}\right) M(\rho)
$$


and

$$
n^{(2)}(\rho, z)=-\frac{\pi^{2} R_{2}{ }^{2} n_{0}}{(2 \pi)^{3}(1+\alpha)^{2}}\left(1+\alpha-\sigma^{2}\right)\left(\mathrm{i} \cos \frac{\Omega_{1} z}{V}-\sin \frac{\Omega_{1} z}{V}\right) M(\rho)
$$

respectively, where

$$
M(\rho)=\int_{0}^{\infty} P\left(k_{\perp}^{2}\right) J_{0}\left(\rho k_{\perp}\right) k_{\perp} \mathrm{d} k_{\perp} .
$$

The numerical results computed for Equations (46), (53) and (56) are shown in Figs. 13-15, respectively.

It is noted that if the magnetic field is so weak such that the ion-cyclotron frequency is much smaller than the ion-acoustic frequency, the magnetic field effect can be ignored in

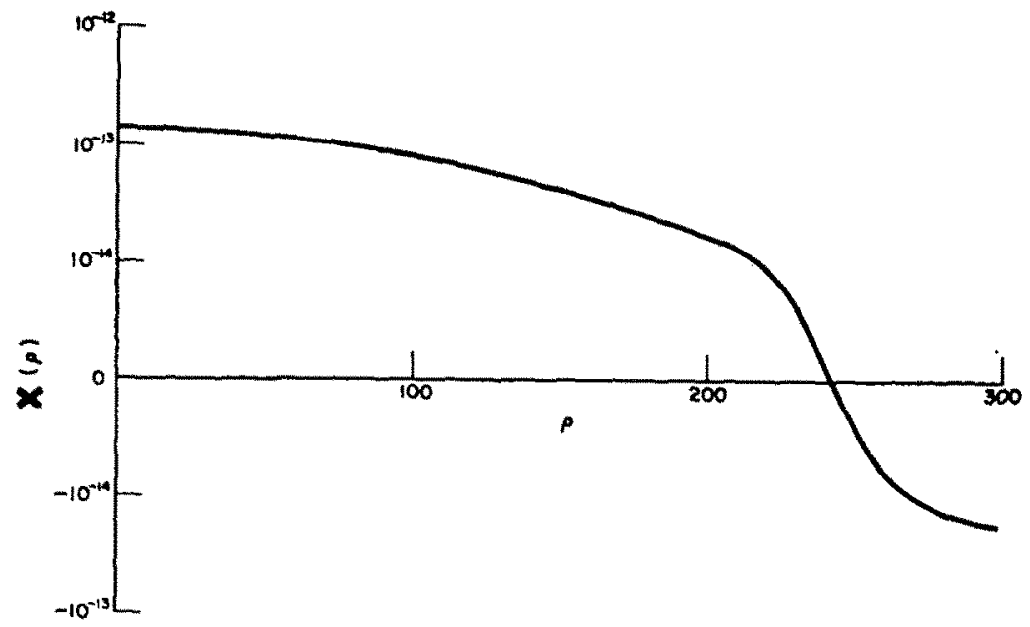

Fro. 13. Function $X$ vs. $P$ (EQuatron 46).

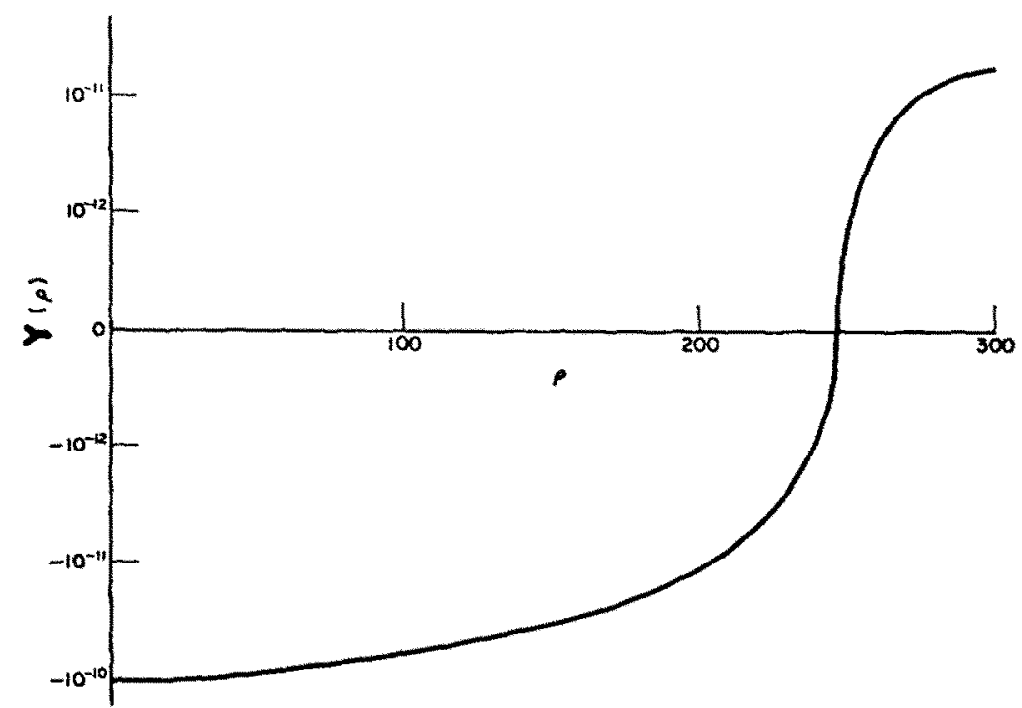

Fro. 14. Function $Y$ vs. $\rho$ (EQUATION 53). 


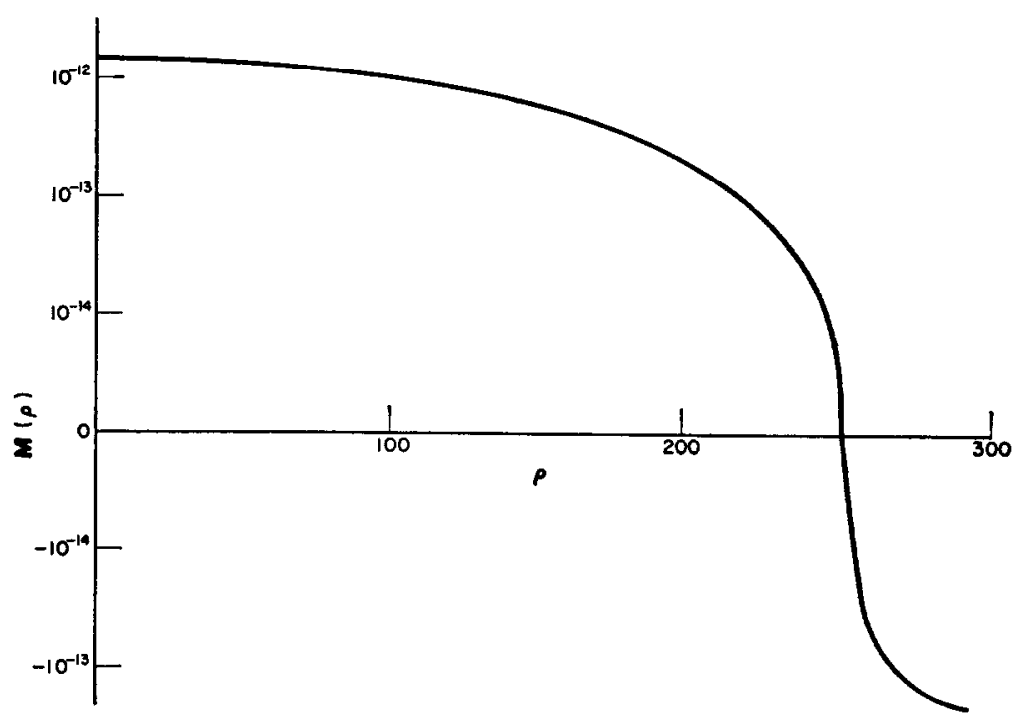

Fig. 15. Function $M$ vs. $\rho$ (Equation 56).

the calculation. On the other hand, if the ion-cyclotron frequency is much greater than the ion-acoustic frequency, we can discuss the results under two different conditions: (1) When the frequency of the perturbed wave, induced by the obstacle, is not close to the ion-cyclotron frequency, then in the $z$-direction we have sinusoidal ion-cyclotron oscillations, and strongly damped motion along $\rho$-direction; (2) When the frequency of the perturbed wave, induced by the obstacle, is very close to the ion-cyclotron frequency, the strong cyclotron damping makes the oscillation die out rapidly along the $z$-direction, and it has, however, relatively less effect on the amplitude of the oscillatory motion along the $\rho$-direction.

\section{CONCLUSION}

The dynamics of interaction between a rarefied plasma and a moving probe, whether it is natural such as a comet or artificial, e.g., a satellite, often plays a vital role in the studies of the upper atmosphere and the interplanetary gas. Sometimes the interaction process can become multifold. The present modest attempt to study this complex particlefield interaction utilizes a simple idealized model to gain some insight in the physics of the problem in question. In order not to make this paper unwieldy, we have not attempted much ramification of the results, e.g., the implication of the multi-lobes in the perturbation density distributions of ions and, of course, electrons (Figs 7-10) to the radar cross section problem.

The surprisingly pronounced effect introduced by the bi-thermal characteristics of the ionosphere upon the plasma tail structure is worth noting both for the single scattering and the double scattering. Should the bi-thermal state $\left(T_{\mathrm{e}} \gg T_{\mathrm{f}}\right)$ be confirmed for the upper ionosphere under direct solar radiation, much of the studies on plasma interactions for gas in thermal equilibrium should be re-evaluated for the cases with the quasi-equilibrium state of $T_{\mathrm{e}} \gg T_{\mathrm{i}}$. In drawing conclusions one, of course, needs to comprehend the basis of comparison of the scattering results with $T_{\mathrm{e}} / T_{\mathrm{i}}=1$ and $T_{\mathrm{e}} / T_{\mathrm{i}} \gg 1$. Note that in the present comparison the speed ratios are made equal, i.e., the ions temperatures in the two states are the same. 
It should be mentioned that the method of treating the double-scattering problem can be extended straightforwardly to the $N$-scattering problems.

Acknowledgement-The present work has been supported by the NASA Research Grant NsG-660.

\section{REFERENCES}

1. BrermanN, L. and Lüst, R. (1964). The Solar Wind (Ed. Mackin, Jr., R. J. and Neugebauer, M.) Pergamon.

2. Lru, V. C. (1967). Nature 215, 217.

3. AL'Pert, YA. L., Gurevich, A. V. and PITAevskm, L. P. (1965). Space Physics with Artificial Satellites. Consultants Bureau, New York.

4. Lru, V. C. and JEw, H. (1966). Proceedings Sth Int. Symp. Rarefied Gas Dy. Oxford (Adv. Appl. Mech. Suppl. 4, Vol. 2, Academic Press (1967), New York).

5. Lru, V. C. (1965). Nature 205, 883.

6. STrx, T. H. (1962). The Theory of Plasma Wates. McGraw-Hill.

7. Drell, S. D., Foley, H. M. and Ruderman, M. A. (1965). J. geophys. Res. 70, 3131.

8. Faddeyeva, V. N. and TERentev, N. M. (1954). Tables of the Probability Integral for Complex Arguments. GITTL, Moscow.

9. LANDAu, L. D. (1946). J. Phys. (USSR) 10, 25.

10. Frred, B. D. and Conte, S. D. (1961). The Plasma Dispersion Function. Academic Press.

\section{APPENDIX}

Consider the following integration

$$
F(\theta, A, \alpha)=\cos \theta \int_{0}^{\pi} \frac{\Phi(A \cos x) \sin x \cos x \mathrm{~d} x}{\left[\cos ^{2} x-\sin ^{2} \theta+2 \mathrm{i} \delta \cos x \cos \theta\right]^{3 / 2}} .
$$

Since $\pi / 2<\theta<3 / 2 \pi$, i.e., $\cos \theta<0$, the sign of the imaginary part in the denominator of Equation (A.1) is negative, it follows (Landau, 1946) that the contour of integration shall be taken along the real axis (see Fig. A).

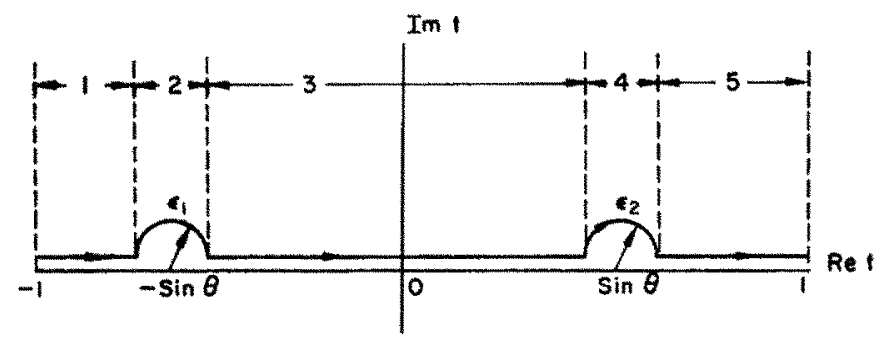

Fig. A. OPEn CONTOUR OF INTEGRation.

Letting $\delta \rightarrow+0$, Equation (A.1) becomes

$$
F(\theta, A, \alpha)=\cos \theta \int_{-1}^{1} \frac{\Phi(A t) t \mathrm{~d} t}{[(t-\sin \theta)(t+\sin \theta)]^{3 / 2}},
$$

where $t=\cos x$.

It is clear that the point of singularities are $\pm \sin \theta$. The contour of integration (Landau, 1946) can be divided into five parts as shown in Fig. A. Investigating Equations (13) and (20), we find that both $\operatorname{Re} \Phi^{(1)}(A t)$ and $\operatorname{Re} \Phi^{(2)}(A t)$ are even functions of $t ; \operatorname{Im} \Phi^{(1)}(A t)$ and $\operatorname{Im} \Phi^{(2)}(A t)$ odd functions of $t$ for the cases of the single and double scattering 
respectively. It follows that

$$
\begin{array}{ll}
\lim _{s_{1}, \varepsilon_{2} \rightarrow 0} & \int_{-\sin \theta+s_{1}}^{\sin \theta-\varepsilon_{3}} \frac{\operatorname{Re} \Phi(A t) t \mathrm{~d} t}{\left(t^{2}-\sin ^{2} \theta\right)^{3 / 2}}=0 \quad \text { along contour (3) } \\
=0 \quad & \lim _{s_{1}, \varepsilon_{2} \rightarrow 0}\left\{\int_{\sin \theta+s_{1}}^{1} \frac{\operatorname{Im} \Phi(A t) t \mathrm{~d} t}{\left(t^{2}-\sin ^{2} \theta\right)^{3 / 2}}-\int_{\sin \theta+\varepsilon_{2}}^{1} \frac{\operatorname{Im} \Phi(A t) t \mathrm{~d} t}{\left(t^{2}-\sin ^{2} \theta\right)^{3 / 2}}\right\} \\
=0 \text { along contour (1) and (5). }
\end{array}
$$

The method of integration by parts is valid only for the integration along the real axis. After applying the integration by parts twice, we obtain

$$
\begin{gathered}
\lim _{\varepsilon_{1}, \varepsilon_{2} \rightarrow 0} \int_{(1),(5)} \frac{\operatorname{Re} \Phi(A t) t \mathrm{~d} t}{\left(t^{2}-\sin ^{2} \theta\right)^{3 / 2}}=\left.\lim _{\varepsilon_{1}, \varepsilon_{2} \rightarrow 0} \frac{-\operatorname{Re} \Phi(A t)}{\left(t^{2}-\sin ^{2} \theta\right)^{1 / 2}}\right|_{(1),(5)}-\operatorname{Re} \Phi^{\prime}(A \sin \theta) \ln (A \sin \theta) \\
\quad+\int_{\sin \theta}^{1} \operatorname{Re} \Phi^{\prime \prime}(A t) \ln \left(t+\sqrt{\left.t^{2}-\sin ^{2} \theta\right)} \mathrm{d} t\right. \\
\quad \text { along contour (1) and (5). } \\
\lim _{\varepsilon_{1}, \varepsilon_{2} \rightarrow 0} \int_{(3)} \frac{\operatorname{Im} \Phi(A t) t \mathrm{~d} t}{\mathrm{i}\left(t^{2}-\sin ^{2} \theta\right)^{3 / 2}}=\lim _{\varepsilon_{1}, s_{2} \rightarrow 0}-\left.\frac{\operatorname{Im} \Phi(A t)}{\mathrm{i}\left(t^{2}-\sin ^{2} \theta\right)^{1 / 2}}\right|_{(3)}-\sin \theta\left[\frac{\pi}{2} \operatorname{Im} \Phi^{\prime}(A \sin \theta)\right] \\
\quad+\sin \theta \int_{0}^{\sin \theta} \operatorname{Im} \Phi^{\prime \prime}(A t) \sin ^{-1}\left(\frac{t}{\sin \theta}\right) \mathrm{d} t \text { along contour (3). }
\end{gathered}
$$

For the contour (2) and (4) we have the results

$$
\begin{array}{ll}
\lim _{\varepsilon_{1} \rightarrow 0} \frac{1}{\sqrt{ } 2 \varepsilon_{1}^{1 / 2} \sin ^{1 / 2} \theta} \Phi(A \sin \theta)(i+1) & \text { for contour (2), } \\
\lim _{e_{2} \rightarrow 0} \frac{1}{\sqrt{ } 2 \varepsilon_{2}^{1 / 2} \sin ^{1 / 2} \theta} \Phi(A \sin \theta)(i+1) & \text { for contour (4) }
\end{array}
$$

respectively. Since

$$
\begin{aligned}
& \left.\lim _{\varepsilon_{1}, \varepsilon_{2} \rightarrow 0} \frac{-\operatorname{Re} \Phi(A t)}{\left(t^{2}-\sin ^{2} \theta\right)^{1 / 2}}\right|_{(1),(5)}=\lim _{\varepsilon_{1}, \varepsilon_{2} \rightarrow 0}\left[-\frac{\operatorname{Re} \Phi(A \sin \theta)}{\sqrt{ } 2 \sin ^{1 / 2} \theta}\left(\frac{1}{\varepsilon_{1}^{1 / 2}}+\frac{1}{\varepsilon_{2}^{1 / 2}}\right)\right] \\
& \left.\lim _{\varepsilon_{1}, \varepsilon_{2} \rightarrow 0} \frac{-\operatorname{Im} \Phi(A t)}{\mathrm{i}\left(t^{2}-\sin ^{2} \theta\right)^{1 / 2}}\right|_{(3)}=\lim _{\varepsilon_{1}, \varepsilon_{2} \rightarrow 0}-\frac{1}{\mathrm{i}}\left[\frac{\operatorname{Im} \Phi(A \sin \theta)}{\sqrt{ } 2 \sin ^{1 / 2} \theta}\left(\frac{1}{\varepsilon_{1}^{1 / 2}}+\frac{1}{\varepsilon_{2}^{1 / 2}}\right)\right] .
\end{aligned}
$$

Combining all these paths of the contour of integration, we have the results as shown in Equations (12) and (19)

where

$$
\begin{aligned}
F(\theta, A, \alpha)=\cos \theta[ & \operatorname{Re} \Phi^{\prime}(A \sin \theta) \ln \sin \theta+\int_{\sin \theta}^{1} \operatorname{Re} \Phi^{\prime \prime}(A t) \ln \left(t+\sqrt{t^{2}-\sin ^{2}}\right) \\
& \left.+\left\{\frac{\pi}{2} \operatorname{Im} \Phi^{\prime}(A \sin \theta)-\int_{0}^{\sin \theta} \operatorname{Im} \Phi^{\prime \prime}(A t) \sin ^{-1}\left(\frac{t}{\sin \theta}\right) \mathrm{d} t\right\}\right]
\end{aligned}
$$

$$
\Phi^{\prime}=\frac{\mathrm{d} \Phi}{\mathrm{d} t} \quad \text { and } \quad \Phi^{\prime \prime}=\frac{\mathrm{d}^{2} \Phi}{\mathrm{d} t^{2}}
$$

